\title{
Touch Affordances
}

\author{
Karin Slegers ${ }^{1}$, Dries De Roeck ${ }^{1}$, and Timo Arnall ${ }^{2}$ \\ ${ }^{1}$ Centre for User Experience Research, Parkstraat 45, 3000 Leuven, Belgium \\ \{karin.slegers, dries.deroeck\} @soc.kuleuven.be \\ ${ }^{2}$ Oslo School of Architecture and Design, Maridalsveien 29, 0175 Oslo, Norway \\ timo.arnalleaho.no
}

\begin{abstract}
The workshop "Touch Affordances" addresses a concept relevant to human computer interactions based on touch. The main topic is the challenge of applying the notion of affordances to domains related to touch interactions (e.g. (multi)touch screens, RFID \& NFC, ubiquitous interfaces). The goals of this workshop are to launch a community of researchers, designers, etc. interested in this topic, to create a common understanding of the field of touch affordances and to generate ideas for new research areas for intuitive touch interactions. The workshop will be highly interactive and will have a creative, generative character.
\end{abstract}

Keywords: Affordances, HCI, intuitive design, NFC, RFID, (multi)touch interface, touch experience, ubiquitous interface.

\section{Introduction}

The focus of this workshop is on possible fields and domains where 'touch' as a means of human computer interaction is, or could be implemented. For the purpose of this workshop, the term "touch interaction" is used to refer not only to (multi)touch screens, but also to RFID and NFC related technologies, to gesture -based interactions and ubiquitous interfaces because these technologies share the basic interactions of touching, grasping, making hand movements etc.

The topic of this workshop is "Touch affordances". While on the one hand touchbased interactions require very simple actions, on the other hand, touch-based interfaces often are unfamiliar to users and therefore do not invite them to perform the correct actions. Findings of previous studies suggest that unfamiliarity with touchrelated interfaces can indeed result in difficulties with regard to interaction (e.g. Belt, Greenblatt, Häkkilä \& Mäkelä, 2006; Peltonen, Kurvinen, Salovaara, et al., 2008). This topic leads to the question of how to design simple yet intuitive touch interfaces.

There have been some initiatives to design intuitive graphics to clarify the intended touch-based interaction to the user (e.g. Arnall, 2006; Saffer, 2008). When talking about "touch affordances" within the context of this workshop, we are looking beyond signage and items on a screen that invite a person to 'touch' it. Instead, touch affordances are envisioned in a much broader way. Areas such as ubiquitous computing, haptics, presence, experience research and human computer confluence play a major role in this. 


\section{The Workshop}

This workshop aims to 1) bring together people who are active in the topic of the workshop to create a community; 2) come to a shared view on the field of Touch Affordances; and 3) generate ideas for new research areas for innovative (and intuitive) touch interfaces and interactions.

The one day workshop will have a creative, generative character. It will consist of four parts described below. During the workshop, observants will live blog about the workshop. A tag cloud will be generated based on these blogs.

Session 1: Keynote \& wrap-up (1.5 hours, plenary session) A keynote presentation will be given by Timo Arnall, who will provide his vision on the topic of the workshop. After the keynote, the organizers of the workshop will give an overview of the submitted position papers.

Session 2: Introduction to creative session \& plenary brainstorm (1 hour, plenary session) Challenges and questions of interest identified in Session 1 will be the starting point for a generative group discussion. The main goal of this discussion will be to set up a number of design scenarios for which touch interfaces will be relevant.

Session 3: Creative session - developing lo-fi touch affordance prototypes $(1.5$ hours, four groups) Participants will be divided into four groups, based on their interest in one of the scenarios identified in Session 2. These groups will each develop a lo-fi prototype of a touch affordance for one of the scenarios. The workshop organizers will provide materials to create such prototypes. Each group will make a visual representation of their prototype.

Session 4: Testing prototypes collaboratively \& creation of poster ( 2 hours, plenary session) Each group presents the prototype that was developed. The intuitiveness of each prototype will be assessed by conducting interaction tests with other workshop participants. Next, the participants will collaborate to create a poster summarizing the main results of the workshop. The poster will include the four prototype representations, as well as a tag cloud based on the bloggers' reports of the workshop. On the poster, the scenarios, the prototypes and each participant will be linked to relevant tags in order to create a visual map of the field of Touch Affordances and its community.

\section{References}

1. Arnall, T.: A graphic language for touch-based interactions. In: Workshop Mobile Interaction with the Real World (MIRW 2006) in conjunction with MobileHCI 2006, Espoo, Finlands (2006)

2. Belt, S., Greenblatt, D., Häkkilä, J., Mäkelä, K.: User Perceptions on Mobile Interaction with Visual and RFID Tags. In: MIRW 2006, Workshop W5 @ MobileHCI 2006, Espoo, Finland (2006)

3. Peltonen, P., Kurvinen, E., Salovaara, A., Jacucci, G., Ilmonen, T., Evans, J., Oulasvirta, A., Saarikko, P.: It's Mine, Don't Touch: Interactions at a Large Multi-Touch Display in a City Centre. In: Proc. CHI 2008, Florence, pp. 1285-1294. ACM Press, New York (2008)

4. Saffer: Designing Gestural Interfaces. O’Reilly Media Inc., Sebastopol (2008) 